\title{
New image analysis method for the estimation of global and spatial changes in fruit microstructure**
}

\author{
Piotr M. Pieczywek ${ }^{*}$, Justyna Cybulska ${ }^{1}$, Barbara Dyki², Dorota Konopacka ${ }^{2}$, \\ Monika Mieszczakowska-Frac ${ }^{2}$, and Artur Zdunek ${ }^{1}$ \\ ${ }^{1}$ Institute of Agrophysics, Polish Academy of Sciences, Doświadczalna 4, 20-290 Lublin, Poland \\ ${ }^{2}$ Research Institute of Horticulture, Pomologiczna 18, 96-100 Skierniewice, Poland
}

Received September 17, 2015; accepted December 7, 2015

\begin{abstract}
A b s t r a c t. A new image analysis method for the spatial characterization of microscopy images of fruit microstructure is proposed in order to analyse the heterogeneous microstructure of unprocessed fruit and the possible inhomogeneous effects of various technological treatments on this microstructure. The microstructure of tissue samples was characterized using the global statistics of size and shape parameters calculated for all visible objects. Global analysis was supported by a novel algorithm that allowed for drawing of the maps of the cell wall fraction from microscopy images and for the analysis of both global and local compaction or loosening of tissue. The spatial distribution of the cell wall fraction was visualised in the convenient form of bivariate histograms. To test the developed image analysis protocols, structural changes resulting from ultrasonic and osmotic treatments of apple tissue samples were studied. Peeled and cored apples were submersed in a liquid medium (distilled water or $60{ }^{\circ} \mathrm{Bx}$ sucrose solution) for 45 and $90 \mathrm{~min}$ with and without ultrasonic treatment. After these treatment procedures, tissue samples were cut into slices, stained and imaged using a microscope. The proposed method allowed to characterise the effects of different sample treatments.

K e y w o r d s: image analysis, apple, microstructure, osmotic, ultrasound, bivariate histograms
\end{abstract}

\section{INTRODUCTION}

Mass transfer through fruit tissue is affected by several aspects of its morphology, such as; the intracellular network of spaces, cell wall properties, boundary (cell walls) fractions and plasmalemma permeability. The presence of intracellular spaces has a significant influence on the physical properties of plant tissue which consists of a significant fraction of pores ranging from $1 \%$ for tissues that con-

*Corresponding author e-mail: p.pieczywek@ipan.lublin.pl **This research had been conducted within the 'BIOSUSZ' PBS Project financed by the National Centre for Research and Development (PBS1/A8/13/2012). tain densely-packed cells of polygonal shapes like potato or carrot parenchyma, to over $30 \%$ in the case of apples (Mebatsion et al., 2009). The percentage volume of pores is associated with firmness and crispness and promotes the texture of mealiness (Harker and Suthrland, 1993). The structure of the material is important for supplying and removing the gases $\left(\mathrm{O}_{2}\right.$ and $\left.\mathrm{CO}_{2}\right)$ required for intracellular respiration (Ho et al., 2009, 2010, 2011; Mebatsion et al., 2008) and it also determines water transport mechanisms (Chiralt and Talens, 2005, Mendoza et al., 2007). Tissue pre-treatments leading to changes in structure may significantly alter the effectiveness of further food processing techniques. Ultrasound can affect any element of the tissue sub-structure ie it can lead to cell-to-cell detachment which increases the space between cells, loosening of cell wall assembly, or it may destroy plasmalemma as well as the tonoplast. Generally, the method of ultrasound (US)assisted extraction of biocomponents from rigid structures involves the use of high frequencies ie up to $500 \mathrm{kHz}$. On the other hand low frequencies of $20-40 \mathrm{kHz}$ are used for flexible materials that are complex, substrate-dependent and generally not well characterized (Rosello-Soto et al., 2015a). Power ultrasound refers to sound waves beyond audible frequency between the range of $20 \mathrm{kHz}$ and $1 \mathrm{MHz}$, which are able to interact with both solid and liquid media. Depending on the wave frequency and the level of energy applied, ultrasound can produce chemical, mechanical or physical changes in food processing and the resulting products (Kentish and Ashokkumar, 2011). When power ultrasound is passed through subjected media sequential compressions and expansions are generated, which in turn leads to acoustic cavitation effects, seen in the form of gas

(C) 2016 Institute of Agrophysics, Polish Academy of Sciences 
bubbles in the liquid medium that can explosively collapse and generate localised pressure as well as increases in temperature (Simal et al., 1998; Wan et al., 1992). Due to its ability to interact with a wide range of media, ultrasound is becoming a regular feature in many aspects of agro-food technology (Cárcel et al., 2012). US treatment is one of those promising non-conventional food processing technologies that is especially suitable for the preservation of the fluid foods. US may be used alone or in combination with other preservation techniques, such as mild heating, high pressures and antimicrobials (Zinoviadou et al., 2015). US treatment is also considered to be one of the most important non-conventional technologies that are being used by different food scientists and industry to improve the recovery rate of high-added value compounds from the different wastes and by-products (Barba et al., 2015a, 2015b; Koubaa et al., 2015; Rosello-Soto et al., 2015b; Šic Žlabur et al., 2015). Ultrasonic pre-treatment offers new possibilities for biological material modification, something which has been thoroughly investigated, with reference to its use as a tool for enhancing biomaterial drying efficiency (García-Pérez et al., 2007; Mothibe et al., 2011). Despite the existence of numerous examples of studies concerning ultrasound-biostructure interactions, the tissue complexity and gaps in methodological aspects still require more detailed study that should confirm the anticipated interaction mechanisms.

The motivation for this study was to show the effect of ultrasonic and osmotic treatments on the microstructure of apples as a model fruit. Since the change of microstructure is anisotropic, future research is needed to develop new methods of image analysis. Therefore, the goal of this work was to develop new image analysis methods for global and local characterisation of fruit tissue structure that would be useful for studying structural changes caused by osmotic treatment.

Two methodologies were applied for the evaluation of structural changes of the tissue-conventional global image analysis and a novel analysis based on the calculation of spatial maps of local variations of tissue structural parameters. The first one involved image segmentation and quantification using a set of geometric parameters calculated for each individual object. The new methodology presented involves calculations of maps of the cell wall fraction representing local variations in microstructure density. Spatial maps were quantified by means of bivariate histograms showing the distribution of the cell wall fraction with respect to the relative position of the sampling area.

\section{MATERIALS AND METHODS}

The common apple variety 'Idared' was used as a raw material. The apples were collected at harvest maturity from the Experimental Orchard of the Research Institute of Horticulture in Dabrowice, Poland. Until it was time for processing, the fruit was stored at regular cold storage conditions $\left(0^{\circ} \mathrm{C}, 90 \% \mathrm{RH}\right)$.
The apples were peeled and cored by hand before being cut into $1 \mathrm{~cm}$ cubes with a shredder (Hällde RG-100, Kista, Sweden), after which they were submersed in a liquid medium (distilled water or $60{ }^{\circ} \mathrm{Bx}$ sucrose solution) with a fruit to liquid medium ratio of 1:4. The treatment was conducted for 45 and $90 \mathrm{~min}$ with and without the application of ultrasound. The samples were sonicated at $40^{\circ} \mathrm{C}$ in beakers placed in a water bath (InterSonic, Olsztyn, Poland) fitted with ultrasonic transducers $\left(25 \mathrm{kHz}, 0.1 \mathrm{~W} \mathrm{~cm}^{-3}\right)$ and a shaking plate ( 30 r.p.m.). After treatment the apple cubes were removed from the beakers, strained and rinsed for a few seconds in tap water, then blotted with absorbent paper to remove any excess solution.

For each type of sample treatment two technical repetitions were carried out. The material obtained was immediately frozen and totally dehydrated using a freeze drying method, this allowed for good structure fixation. As a reference material freshly cut apple cubes were frozen and freeze dried without any treatment. Freeze drying was carried out using a standard procedure - samples were frozen using liquid nitrogen and placed in the vacuum chamber of a lyophiliser. Finally 9 objects with different tissue structure modifications induced by various technical treatments, with and without the application of ultrasound were generated.

For histological (planimetric) studies fragments of freeze dried apples of diversified tissue structure were obtained using the procedure described above. The microscopy samples were prepared using the standard paraffin method. The cubes of apples tissue were fixed with CrAF (chromic acid, acetic acid, formalin), dehydrated in ethanol and embedded in paraffin. Material hardened in paraffin was cut into slices using a rotary microtome (PFM Medical Rotary 3002), each slice had a thickness of $15 \mu \mathrm{m}$, and then the slices were stained with safranin and fast green for light microscopy analysis (Gerlach, 1972).

For the analysis of global geometrical descriptors of tissue microstructure, images from an optical transmission microscope were used. Images were captured using an OLYMPUS BX51 (Olympus Corporation, Tokyo, Japan) microscope, equipped with UPlanSApo $4 \mathrm{x} / 0.16$ eyepiece with $4 x$ magnification. Images were recorded with a resolution of 1024 pixels. The pixel size was $3.14 \mu \mathrm{m}$. For each treatment, 10 to 20 microscopy images were recorded. The images were segmented using a proprietary code developed in Matlab (MathWorks, Natick, MA, USA) and the image analysis tool pack. Due to vignetting of the optical system all images were pre-processed using the illumination field flattening procedure. The contrast and clarity of the images were enhanced by consecutive Top-hat and Bottomhat operations. Subsequently, a median filter was applied in order to remove noise from the images and to smooth the contours of the objects. Segmentation was carried out using the standard binary thresholding method. The threshold value was arbitrarily chosen and it remained constant 


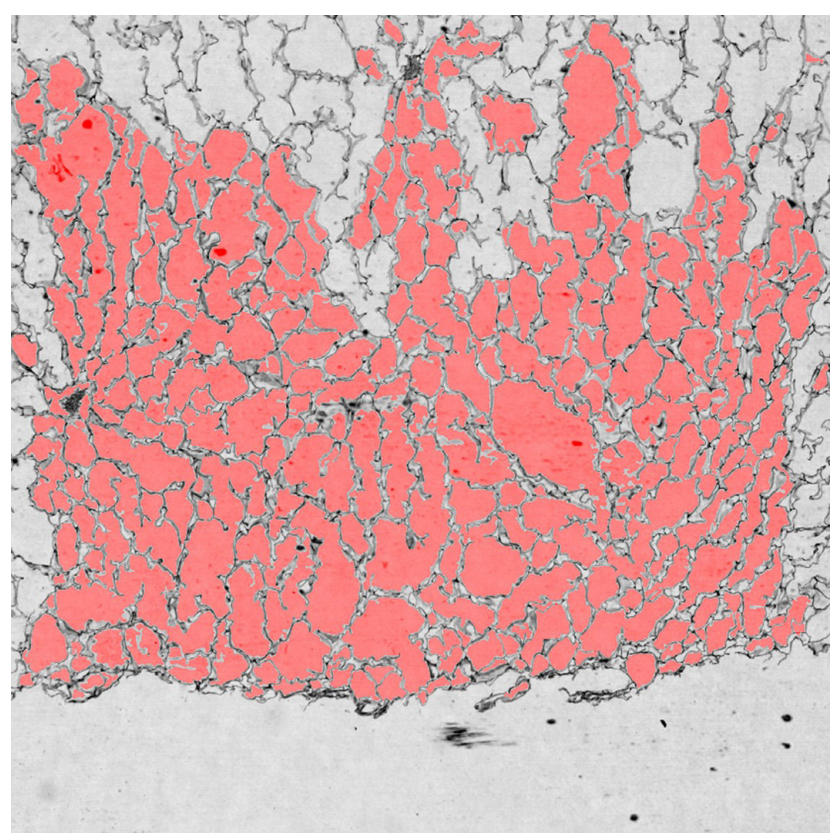

Fig. 1. Example of apple structure and the results of image analysis - all separated, non-boundary objects are marked in red.

for all images. Partially separated objects were merged by means of consecutive morphological operations - dilatation and erosion (Fig. 1).

All boundary objects as well as objects consisting of less than 100 pixels were excluded from the analysis. Statistical analysis was carried out on representative samples of 1050 objects randomly selected for each treatment. Furthermore no distinction between cells and intracellular spaces was made. All objects were analyzed without being divided into categories.

Each selected object was described by four geometrical descriptors: area, perimeter, elongation, and circularity that were calculated using standard MATLAB protocols.

In order to obtain maps of the cell wall fraction from the cross-sections of the sample, images were captured using an Olympus SZX16 microscope with SDF PLAPO 1.6XPF objective. Images were recorded with a Canon Power Shot A640 digital camera. Initial imaging resolution was equal to $1.93 \mu \mathrm{m}$ per pixel. Images used for cell wall fraction mapping were composed of 4-5 smaller pictures of tissue samples. Due to long processing times, the final mosaic images were scaled down to $25 \%$ of their initial size (8.6 - 9.0 mega pixels). Images were recorded in transmission mode.

Images of apple tissue samples were captured in RGB colour space and initially de-noised with a $3 \times 3$ median filter. The contrast and clarity of the images were enhanced by consecutive top-hat and bottom-hat operations. Contrast enhancement was achieved by subtraction of the image resulting from the bottom-hat operation from the sum of the original image and the result of the top-hat operation.
Application of the illumination field-flattening procedure was required, because the optical system produced a relatively strong effect of vignetting. The non-uniformity of field illumination was reduced by the subtraction of the background image from each individual channel. The background image was obtained from the morphological opening of the complement of the original image, using a relatively large structural element in the shape of disk.

The image segmentation procedure was based on colour information stored in CIE L*a*b* colour space. Using the CIE $\mathrm{L}^{*} \mathrm{a} * \mathrm{~b} *$ colour space transformation, the image was divided into three components - the lightness $\mathrm{L}^{*}$ component and two colour components a* (green-magenta) and $b^{*}$ (blue-yellow). The colour transformation was carried out using built-in Matlab protocols described in the Mathlab Reference Guide (Mathworks, 2011). The following segmentation was based on two colour components $a^{*}$ and $b^{*}$, which served as the input data for the k-means clustering algorithm. Clustering was carried out using the squared Euclidean distance as a measure of the distance between cluster centroids. The clustering algorithm was run ten times with the assumption that three clusters were present in the image data. The iteration of the lowest within-cluster sums of point-to-centroid distances was chosen as the final result. It was assumed that the pixels of the biggest cluster represented the image background, while the sum of pixels of the two remaining clusters indicated cell walls. On this basis cluster data was converted into binary images of cell walls (Fig. 2a).

All objects consisting of less than 50 pixels were removed from the binary image. Next, the morphological closing function was applied, and all holes visible on the binary image were filled. Holes were defined as a set of background pixels that could not be reached by filling in the background from the edge of the image. The final binary image showed the silhouette of the whole tissue sample (Fig. 2b).

The original binary image of cell walls and the silhouette image were convolved using a mask image in shape of a circle. The values of all the pixels of the mask image inside the circle were equal to one; therefore convolution was computed as a cumulative sum of pixel values that overlapped non-zero elements of the mask image.

Next, the convolved cell wall image was divided pixelwise by the convolved silhouette image. As a result of this operation local values of cell wall fractions (Fig. 2d) were calculated with respect to the pixel neighbourhood defined by the radius of the circle of the binary mask image. To enhance the local variations of the cell wall fraction the global trend of spatial data (Fig. 2e) was estimated and subtracted from each map. The global trend of data distribution was approximated by fitting of the plane equation to cell wall fraction data. The final image (Fig. 2f) shows a map of the cell wall fraction after subtraction of the global trend of data distribution. 


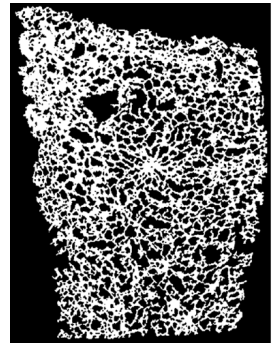

d

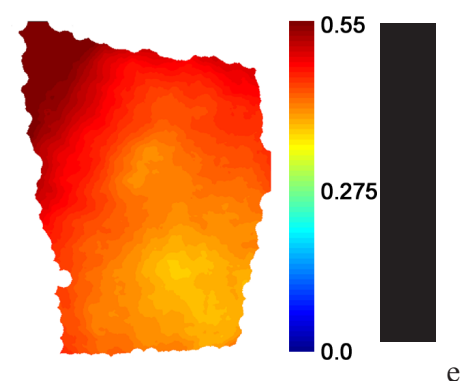

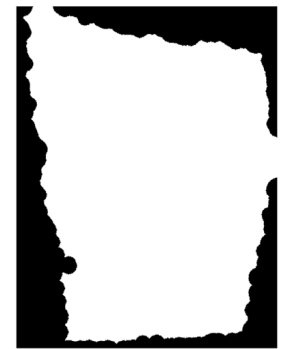

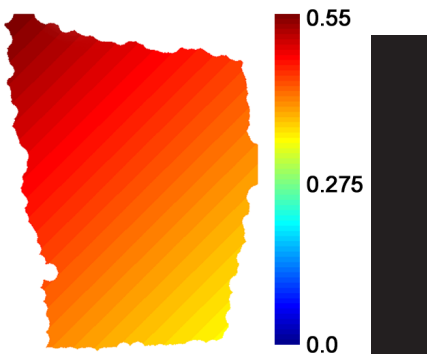

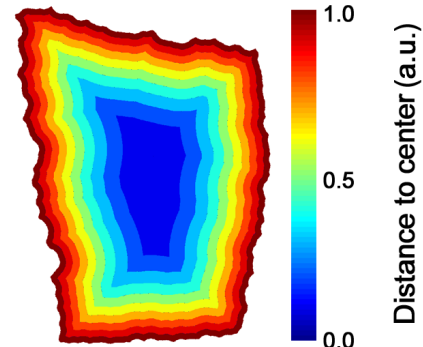

c

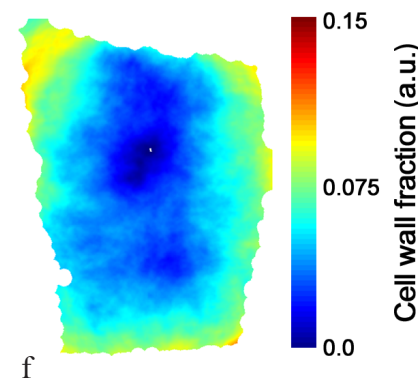

Fig. 2. Image processing steps for the evaluation of the spatial distribution of the cell wall fraction: a - binary image of cell walls, $\mathrm{b}$ - binary silhouette image, $\mathrm{c}$ - pixel distance map, divided into ten equally sized sections, $\mathrm{d}$ - absolute cell wall fraction map, $\mathrm{e}-$ global trend of the cell wall fraction, $\mathrm{f}$ - map of the cell wall fraction after subtraction of the global trend.

a
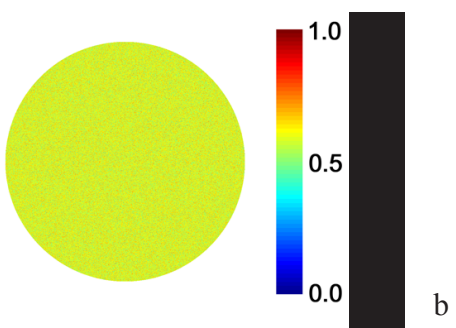

b

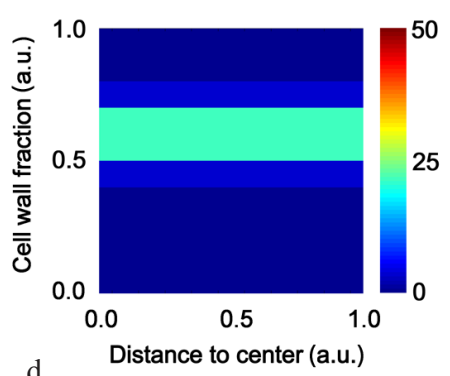

$\mathrm{d}$
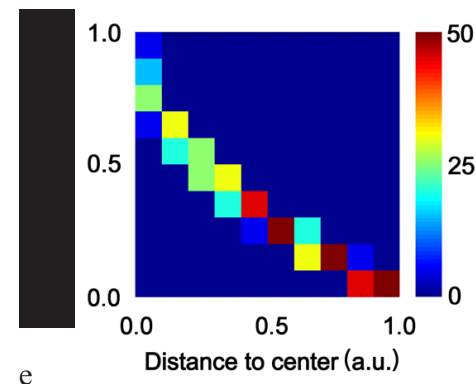
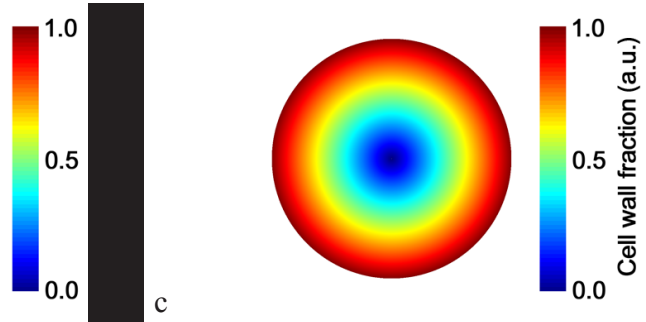

Fig. 3. The bivariate histogram patterns shown as an example of model data: a and d-an example of an artificial sample with Gaussian distribution of the cell wall fraction and corresponding bivariate histogram, b and e - an example of artificial sample with cell wall fraction gradually descending towards the edges of silhouette, $\mathrm{c}$ and $\mathrm{f}$ - an example of artificial sample with cell wall fraction gradually ascending towards the edges of silhouette.

Finally spatial distribution of the cell wall fraction in examined samples was quantified using bivariate histograms. Histograms showed frequencies of co-occurrences of values of the two analyzed variables - cell wall fraction and pixel distance from the sample centre. Histograms were in a shape of two dimensional $10 \times 10$ matrices with rows corresponding to cell wall fraction intervals and columns corresponding to intervals of normalised distance, both

organised in ascending order with the origin at the bottom left corner of the matrix. Each entry of this matrix indicated the number of occurrences of the cell wall fraction at a certain distance that falls into a specific combination of intervals.

The spatial position of pixels expressed as the distance from the sample centre was calculated using the Euclidean distance transform of the binary silhouette image. Distance 
values were scaled to fit the $0-1$ range (Fig. 2c) and divided into ten intervals that occupied the same area of the distance map. For each interval, a histogram of cell wall fraction values was calculated and used as columns of bivariate histogram matrix.

In order to investigate the behaviour of bivariate histograms three artificial types of cell wall fraction distribution were generated (Fig. 3a, b, and c) and tested on the example of a circle shaped sample. Figure 3 a depicts the case of uniform distribution of the cell wall fraction, which is shown in the corresponding bivariate histogram (Fig. 3d). In the case of other two examples spatial trends are present $i e$ the values are descending (Fig. 3b, e) and ascending (Fig. 3c, f) towards the edge of the sample. Bivariate histograms (Fig. 3d, e, and f) should be interpreted in the following way:

- in the case of uniformly distributed cell wall fraction over the whole area of sample the entries of the histogram matrix have similar values and are organised in horizontal direction (Fig. 3d);

- when the cell wall fraction changes with distance, the histogram data is organised in diagonal directions, with slope depending on the rate and direction of the change of cell wall fraction;

- when cell wall fraction values decrease towards the edge of the sample the slope has negative value (Fig. 3e);

- for samples for which cell wall fraction increases along with the distance from the sample centre the slope has positive value (Fig. 3f).

The statistical analysis of global descriptors was carried out using Statistica 10 software (StatSoft, Inc., Tulsa, USA). Differences between micromechanical features of samples subjected to different treatments were analyzed using a one-way ANOVA and post-hoc Tukey HSD test at $\mathrm{p}=0.05$.

\section{RESULTS AND DISCUSSION}

At the present time image analysis is a well-developed method allowing relatively easy extraction of quantitative data from microscopy images (Pieczywek and Zdunek, 2012). Therefore, within the framework of this study, a new method has been developed that allows maps of cell wall fraction to be drawn from microscopy images and moreover it allows analysis of both global and local compaction or loosening of tissue. The method is validated with the images of apple tissue subjected to different pre-drying treatments, thus varying the extent of cellular structure modifications. The objects were preserved using standard fixation and preparation methods, and then observed with an optical transmission microscope, so the procedure could be easily adapted to any other experimental protocol.

The representative images of apple tissue structure, obtained after the applied treatments, are presented in Fig. 4. As it is visually apparent, exposure to both kinds of liquid media and US assistance, induced large modifications in size and shape parameters of the observed microscopic objects. The graphical representation of four geometrical descriptors of microstructure is shown in Fig. 5. Unfortunately, due to wide range of values and high skewness of data distribution, the one-way ANOVA analysis was unable to provide perfectly clear evidence for statistical difference between each group of samples. To be able to analyse the extent of the observed structure changes more thoroughly, the detailed data on the geometric parameters of all the samples, including statistical information, is summarised in Table 1.

In general, the process of apple cubes submersion in a water bath without ultrasound assistance led to an increase in object areas (Fig. 5a). The increase in number of large size objects was indicated by higher maximum values and lower medians compared to control sample (Table 1). a

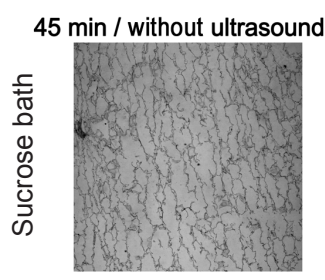

e

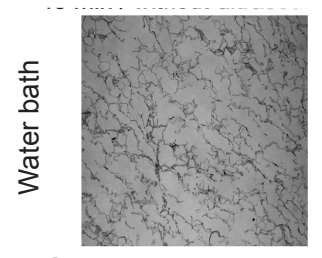

b

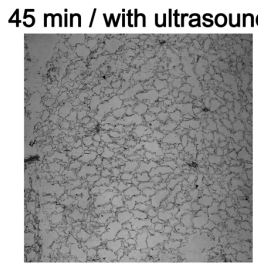

f

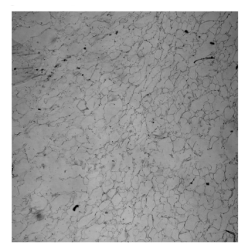

c

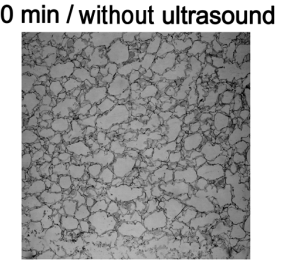

g

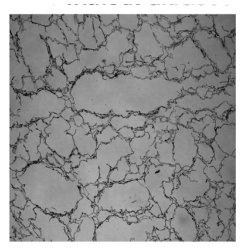

d

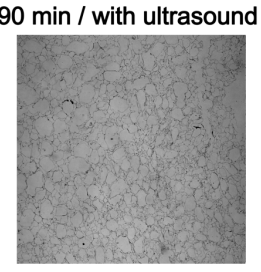

$\mathrm{h}$

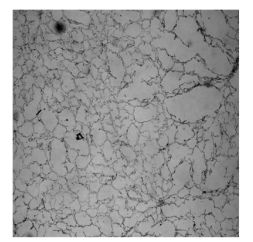

Fig. 4. Microscopy images of apple parenchyma tissue after submersing in: a, b, c, d $-60^{\circ}$ Brix sucrose; e, f, g, h-distilled water; with and without ultrasonic treatment. 


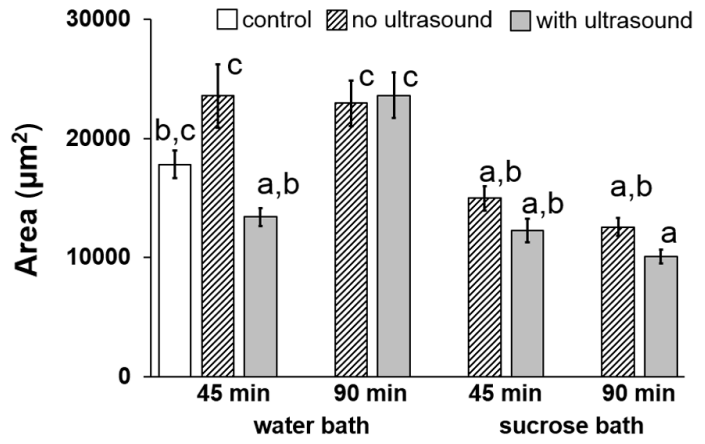

a

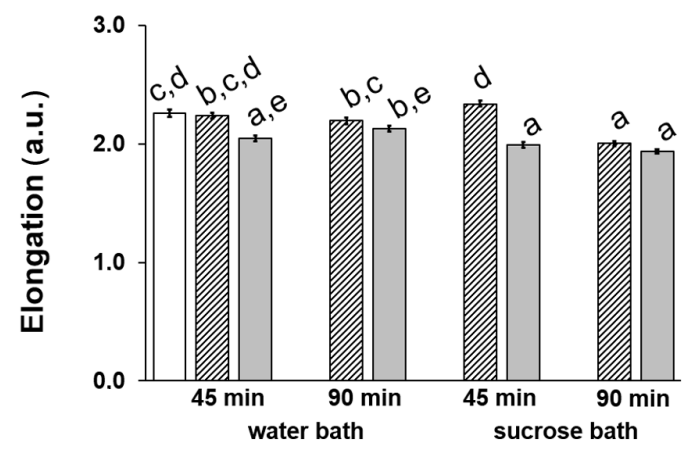

C

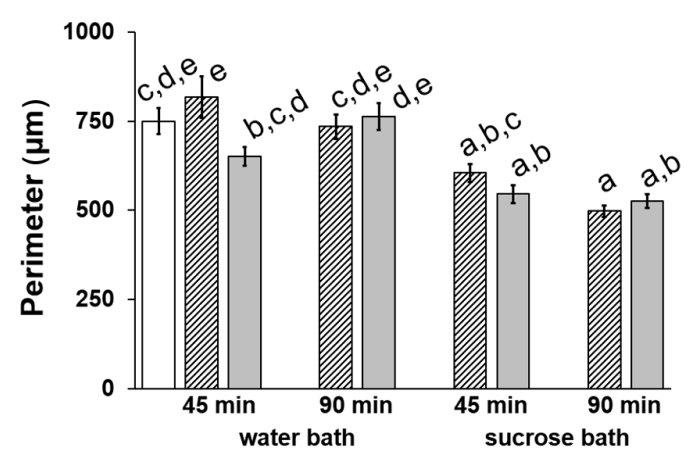

b

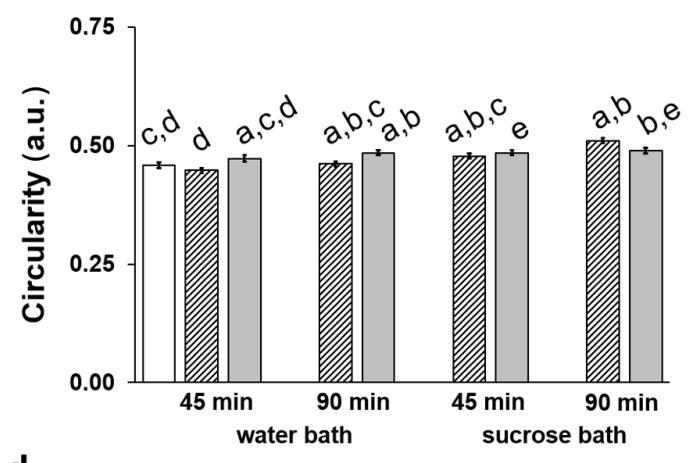

Fig. 5. Results of ANOVA - HSD Tukey test for: $\mathrm{a}$ - area, b - perimeter, c - elongation, and d - circularity of objects from microscopy images. Bars indicate mean values of measured parameters while whiskers show confidence intervals. Letter indexes indicate membership to homogeneous groups (according to ANOVA with $\mathrm{p}=0.05$ ).

Also, slight decrease in mean, median and maximum area of objects was observed when the treatment duration was extended. However statistical analysis showed no significant difference between control sample and the samples treated for 45 and 90 min (Fig. 5a). Similar trends as for object areas were observed for perimeter values.

In the case, when the apple tissue was immersed in a water bath, but the treatment was combined with the application of the ultrasound, the opposite effect was observed. First, a sample immersed in distilled water for 45 min displayed slight decrease in object areas in comparison to the control sample, however, when the treatment duration was extended to $90 \mathrm{~min}$, large and statistically significant increase in mean, median and maximum of object areas was observed. As before, similar conclusions applied to the changes in perimeters of objects. To summarise, the immersion of apple cubes in distilled water carried out for $90 \mathrm{~min}$, with and without the application of ultrasonic waves, caused expansion of tissue structure and increase in size parameters (area and perimeter).

In contrast to the immersion of the apple cubes in distilled water, their osmotic treatment in sucrose solution (sucrose bath) led to the contraction of the structure, resulting in decrease of both size parameters discussed above (Fig. 4a, b). Moreover, the longer the treatment in sucrose solution was, the greater contraction effect was observed.
The contraction of the tissue structure was intensified when the sample processing was enhanced by ultrasonic treatment. The phenomena of mass exchange intensification observed for US-treated fruit and vegetable tissue is attributed to changes in microstructure, especially to the creation of microscopic channels that favour easier moisture removal (Garcia-Perez et al., 2006). Statistically significant decreases were reported, both for object area, as well as for the perimeter, (Fig. 5a, b).

Regarding the other shape parameters, submersion of apple cubes in a water bath resulted in a decrease of elongation of objects (average, maximum and median) (Table 1, Fig. 5c). After $90 \mathrm{~min}$ in water bath, with and without ultrasonic treatment, the average elongation of objects was lower when compared to the control sample. With the US treatment the average elongation was slightly lower; however, the difference between both treatments was not statistically significant. With ultrasound- aided treatment, the decrease was statistically significant after just $45 \mathrm{~min}$.

For samples treated in a sucrose bath, the elongation of objects decreased with the duration of this process. After $90 \mathrm{~min}$ with and without ultrasonic treatment the average elongation of objects was lower in comparison to the control sample (Fig. 5c - sucrose bath). It should be noted that when ultrasound was applied, significant changes in elongation were observed after $45 \mathrm{~min}$. Longer treatment 
T a b l e 1. Mean values and standard deviations of the measured geometrical descriptors for all treatments

\begin{tabular}{|c|c|c|c|c|c|c|c|c|}
\hline Parameter & Medium & $\begin{array}{c}\text { Ultrasound } \\
\text { treatment }\end{array}$ & $\begin{array}{c}\text { Duration } \\
\text { (min) }\end{array}$ & Mean & $\begin{array}{c}\text { Standard } \\
\text { dev. }\end{array}$ & Minimum & Median & Maximum \\
\hline \multirow{9}{*}{$\begin{array}{l}\text { Area } \\
\left(\mu \mathrm{m}^{2}\right)\end{array}$} & \multirow{4}{*}{ Water } & \multirow[t]{2}{*}{ No } & 45 & 23580 & 91257 & 1163 & 5151 & 1770832 \\
\hline & & & 90 & 22954 & 66580 & 1163 & 4616 & 1156554 \\
\hline & & \multirow[t]{2}{*}{ Yes } & 45 & 13420 & 26666 & 1163 & 4634 & 379355 \\
\hline & & & 90 & 23610 & 65536 & 1163 & 5994 & 965109 \\
\hline & \multirow{4}{*}{ Sucrose } & \multirow[t]{2}{*}{ No } & 45 & 14964 & 35141 & 1163 & 4686 & 527834 \\
\hline & & & 90 & 12558 & 25560 & 1163 & 4052 & 624441 \\
\hline & & \multirow[t]{2}{*}{ Yes } & 45 & 12269 & 33820 & 1163 & 4000 & 808909 \\
\hline & & & 90 & 10112 & 19719 & 1163 & 4000 & 354134 \\
\hline & Control & No & 0 & 17814 & 40364 & 1163 & 5744 & 715582 \\
\hline \multirow{9}{*}{$\begin{array}{l}\text { Perimeter } \\
\quad(\mu \mathrm{m})\end{array}$} & \multirow{4}{*}{ Water } & \multirow[t]{2}{*}{ No } & 45 & 818 & 2021 & 126 & 379 & 37617 \\
\hline & & & 90 & 735 & 1166 & 123 & 365 & 12906 \\
\hline & & \multirow[t]{2}{*}{ Yes } & 45 & 653 & 913 & 122 & 354 & 9202 \\
\hline & & & 90 & 764 & 1296 & 127 & 384 & 18422 \\
\hline & \multirow{4}{*}{ Sucrose } & \multirow[t]{2}{*}{ No } & 45 & 605 & 887 & 126 & 352 & 13221 \\
\hline & & & 90 & 498 & 570 & 126 & 330 & 14086 \\
\hline & & \multirow[t]{2}{*}{ Yes } & 45 & 546 & 880 & 127 & 339 & 17224 \\
\hline & & & 90 & 527 & 673 & 126 & 329 & 9337 \\
\hline & Control & No & 0 & 750 & 1269 & 124 & 402 & 22532 \\
\hline \multirow{9}{*}{$\begin{array}{l}\text { Elongation } \\
\text { (a.u.) }\end{array}$} & \multirow{4}{*}{ Water } & \multirow[t]{2}{*}{ No } & 45 & 2.24 & 0.97 & 1.03 & 2.01 & 8.85 \\
\hline & & & 90 & 2.19 & 1.00 & 1.03 & 1.95 & 9.11 \\
\hline & & \multirow[t]{2}{*}{ Yes } & 45 & 2.05 & 0.81 & 1.03 & 1.82 & 7.15 \\
\hline & & & 90 & 2.13 & 0.85 & 1.04 & 1.88 & 7.12 \\
\hline & \multirow{4}{*}{ Sucrose } & \multirow[t]{2}{*}{ No } & 45 & 2.34 & 1.06 & 1.01 & 2.07 & 8.88 \\
\hline & & & 90 & 2.00 & 0.75 & 1.01 & 1.83 & 6.45 \\
\hline & & \multirow[t]{2}{*}{ Yes } & 45 & 1.99 & 0.75 & 1.02 & 1.81 & 6.14 \\
\hline & & & 90 & 1.94 & 0.68 & 1.02 & 1.77 & 5.55 \\
\hline & Control & No & 0 & 2.26 & 1.05 & 1.01 & 1.97 & 9.45 \\
\hline \multirow{9}{*}{$\begin{array}{l}\text { Circularity } \\
\text { (a.u.) }\end{array}$} & \multirow{4}{*}{ Water } & \multirow[t]{2}{*}{ No } & 45 & 0.45 & 0.19 & 0.01 & 0.44 & 0.97 \\
\hline & & & 90 & 0.46 & 0.19 & 0.03 & 0.47 & 0.99 \\
\hline & & \multirow[t]{2}{*}{ Yes } & 45 & 0.47 & 0.22 & 0.03 & 0.46 & 0.99 \\
\hline & & & 90 & 0.49 & 0.19 & 0.03 & 0.49 & 0.99 \\
\hline & \multirow{4}{*}{ Sucrose } & No & 45 & 0.48 & 0.18 & 0.04 & 0.47 & 0.98 \\
\hline & & & 90 & 0.51 & 0.17 & 0.04 & 0.50 & 0.99 \\
\hline & & Yes & 45 & 0.48 & 0.18 & 0.02 & 0.48 & 0.96 \\
\hline & & & 90 & 0.49 & 0.21 & 0.05 & 0.49 & 0.99 \\
\hline & Control & No & 0 & 0.46 & 0.20 & 0.02 & 0.46 & 0.97 \\
\hline
\end{tabular}




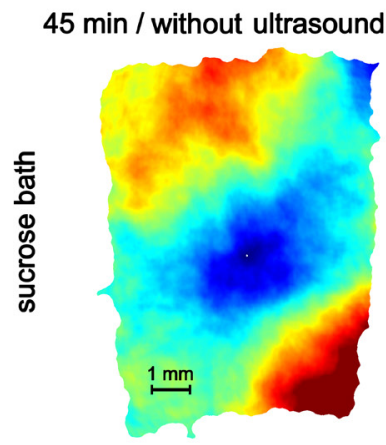

a

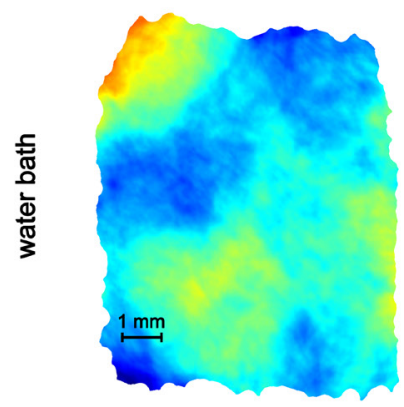

e

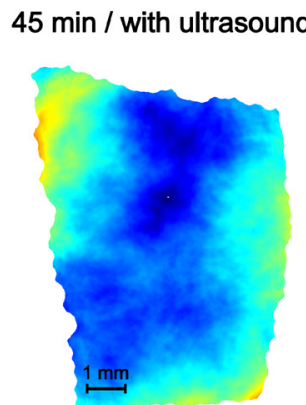

b

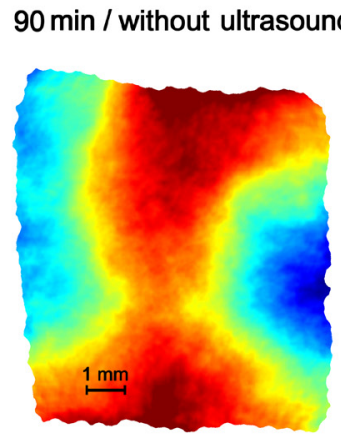

c

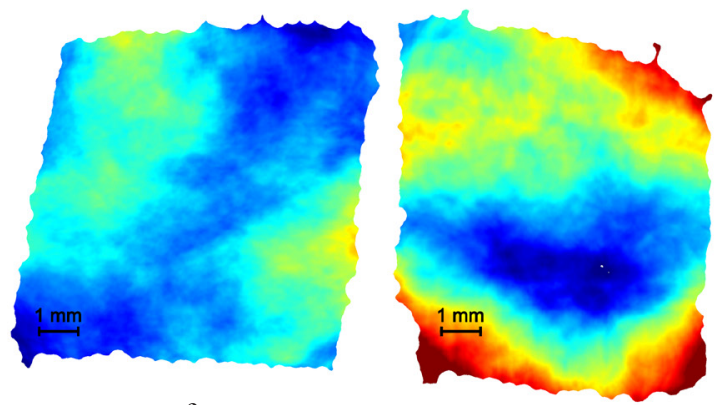

g
$90 \mathrm{~min} /$ with ultrasound

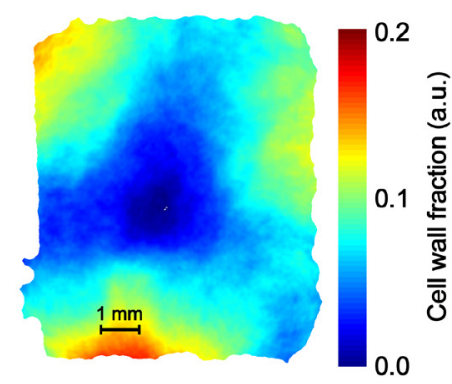

d

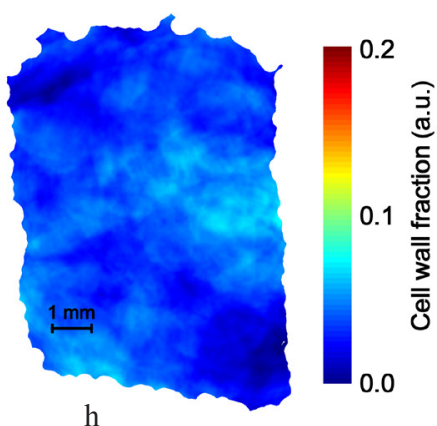

h

Fig. 6. Maps showing distribution of relative cell wall fraction for tissue samples after submersing (45 or 90 min): a, b, c, d-in sucrose $60^{\circ}$ Brix; e, f, g, h-distilled water; with and or without ultrasound treatment. False colours indicate the range of the cell wall fraction from 0 to 0.2 .
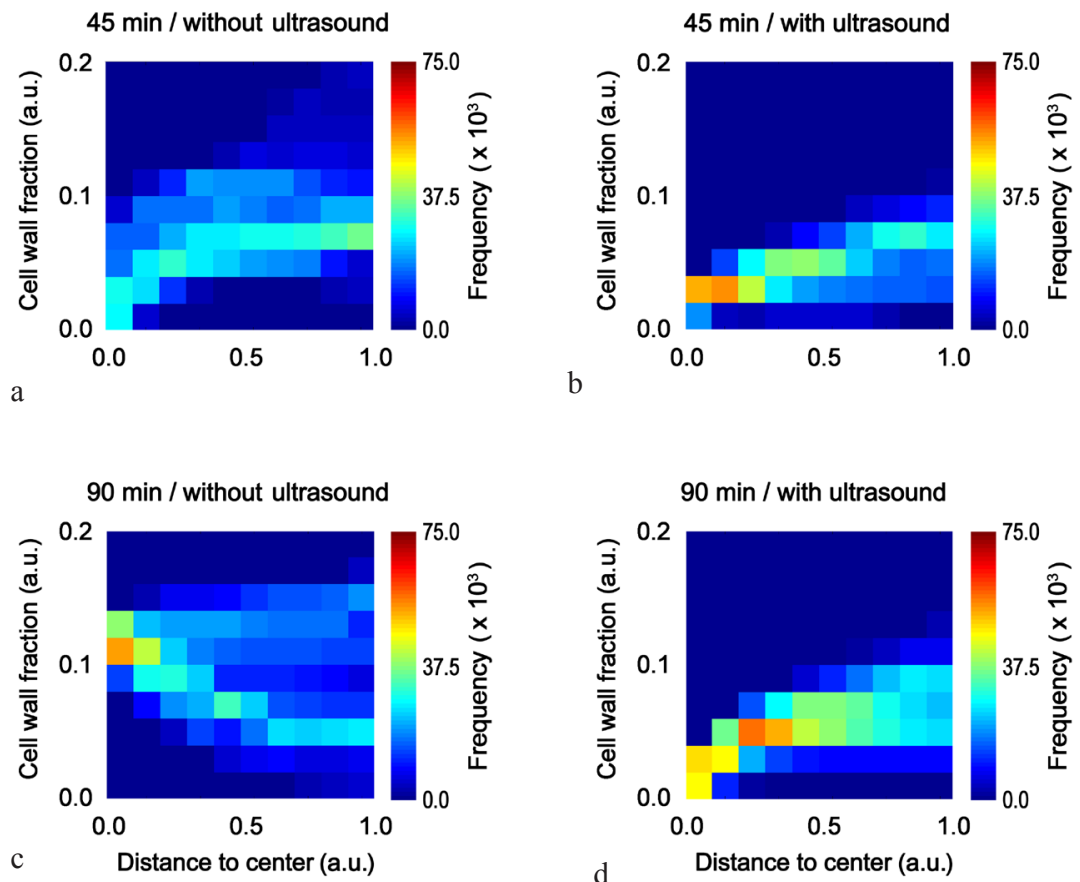

Fig. 7. Bivariate histograms showing the distribution of relative cell wall fraction with respect to spatial position for tissue samples dehydrated in sucrose, visualised in normalised false colours scale: $\mathrm{a}$ and $\mathrm{b}-45 \mathrm{~min}$ treatment without and with US respectively, $\mathrm{c}$ and $\mathrm{d}-90$ min treatment without and with US respectively. 

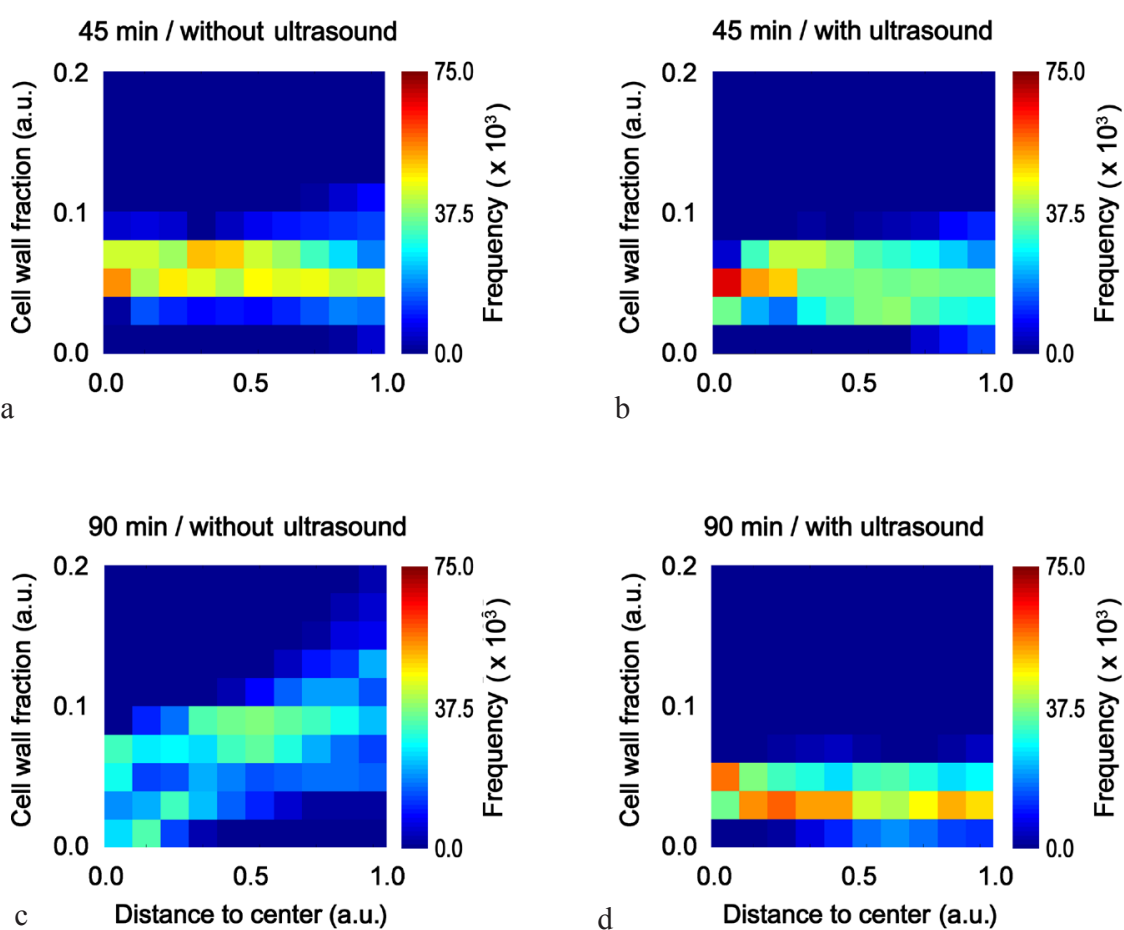

Fig. 8. Bivariate histograms showing the distribution of relative cell wall fraction with respect to spatial position for tissue samples dehydrated in water, visualised in normalised false colours scale: $a$ and b-45 min treatment without and with US respectively, $c$ and d - 90 min treatment without and with US respectively.

resulted in a further slight decrease in elongation, however no significant difference between samples treated for 45 and 90 min was observed.

Regarding object circularity, the same trend was observed - the treatment time elongation favoured an increase in the average circularity value for all the treatments. After $90 \mathrm{~min}$ for almost all applied treatments the observed increase, even though relatively small, was statistically significant.

As already mentioned, despite distinct structural modifications, clearly noticeable in images presented in Fig. 4, the classical analysis of global descriptors (Fig. 5) did not allow a satisfactory quantification of the structural changes. This is indeed due to possibility of heterogeneous original structure and a possibility of inhomogeneous effect of the treatment on the tissue (Legland et al., 2012). This would be the case particularly for dehydrated fruit tissue that may not shrink in a uniform manner (Lewicki and PawlakPorzecka, 2005). Thus, the urgent reason for new analytical approach emerged.

The new method of image analysis allows capture and quantification of local variations in cell wall fraction for each individual sample, furthermore, due to the proposed approach, certain distribution patterns can be quantified by analysing the structure of the bivariate histograms. Since quantification of the spatial data is not a straightforward task at the present time, development of a new method of spatial image analysis to obtain a map of structure descriptors is indispensable for understanding the efficiency of any technologically important treatments (Cantrea et al., 2014; Nguyen et al., 2014; Brosnan and Sun, 2004). Quantified maps of the structure are dependent on the technological parameters of the treatment and in this way they may provide a feedback for the optimization of the technology. Based on a visual analysis of maps of cell wall fraction (Fig. 6) and the relevant bivariate histograms (Figs 7 and 8 ) it is possible to characterise the effects of different sample treatments.

In comparison to samples submersed in sucrose and water without ultrasonic treatment, the cell wall fraction of US-treated samples was lower on average and showed less variation in global values. Also, the rate of change with respect to spatial coordinates was lower for these samples. This was indicated by alignment of the data along lines shifted towards lower values of relative cell wall fraction and by lower slopes and lower vertical spread of values of bivariate histograms for samples treated with ultrasounds (Fig. 7b, d and Fig. 8b, d). All samples dehydrated with sucrose showed some spatial dependency on cell wall fraction values. In general, the cell wall fraction increased with the distance from the sample centre. However, after the ultrasonic treatment the difference in values between points from the edge and the centre of the sample was lower (Fig. 7a, b). Samples treated with ultrasound also showed lower spread of values in vertical direction of the bivariate histogram. This property of histogram can be interpreted as an indicator of directional homogeneity of spatial trend. Values concentrated in narrow ranges shows that spatial 
changes occur in all directions in a similar manner. In the case of a sucrose bath immersion without ultrasonic treatment spatial changes were much less homogenous, which was indicated by wider spread of histogram values (Fig. 7a, c). Moreover, for $90 \mathrm{~min}$ of sample treatment in a sucrose bath without ultrasound assistance it was also possible to quantify strong directional trend which is shown on the histogram as bimodal distribution, with entries concentrated around two separate average values (Figs 6c and 7c).

The samples submersed in water showed more uniform structure without direct spatial trends, this was indicated by horizontal alignment of histogram values (Fig. 8). Also, all these samples had lower absolute values of cell wall fraction than samples dehydrated in sucrose (data not shown) which is perfectly related to differences visible in object areas from microscopic analysis (Fig. 4).

\section{CONCLUSIONS}

1. The novel method of image analysis with respect to spatial distribution of measured parameters brought satisfactory quantification of distinct tissue structure modifications after various treatments were applied. Results showed that the cell wall fraction of samples exposed to ultrasonic waves was lower in average and showed less variation in global values. Also, the rate of change with respect to spatial coordinates was lower for US-treated sample. This leads to a conclusion that ultrasonic treatment might have a positive effect on the mass and energy transfer, which resulted in more homogenous structure of tissue.

2. The data compaction and a convenient way of presenting of the data offer variety of possible applications of presented algorithm. It should be noted, that spatial position of the pixels expressed as the distance from the sample centre is best suited for characterisation of the samples with regular, convex boundaries (circular, rectangular or polygonal). This limitation does not apply to other spatial descriptors. Also, parameters, other than cell wall fraction can be quantified, depending on the type of sample. The bivariate histograms can be employed to describe the distribution of cells shape parameters for instance.

3. Data representation in a form of normalised bivariate histograms is suitable for classification and recognition algorithms. Spatial patterns can be associated with desired structural composition and thus indicate the efficiency of processing applied to a sample. The method opens the possibility of designing of new structure descriptors for understanding the efficiency of any technologically important treatments.

\section{REFERENCES}

Barba F.J., Brianceau S., Turk M., Boussetta N., and Vorobiev E., 2015a. Effect of alternative physical treatments (ultrasounds, pulsed electric fields, and high-voltage electrical discharges) on selective recovery of bio-compounds from fermented grape pomace. Food Bioprocess Technol., 8, 1139-1148.
Barba F.J., Galanakis C.M., Esteve M.J., Frigola A., and Vorobiev E., 2015b. Potential use of pulsed electric technologies and ultrasounds to improve the recovery of highadded value compounds from blackberries. J. Food Eng., 167, 38-44.

Brosnan T. and Sun D.-W., 2004. Improving quality inspection of food products by computer vision - a review. J. Food Eng., 61, 3-16.

Cantrea D., Eastb A., Verboven P., Arayab X.T., Herremansa E., Nicolaïa B.M., Pranamornkithb T., Lohc M., Mowatd A., and Heyesb J., 2014. Microstructural characterisation of commercial kiwifruit cultivars using X-ray micro computed tomography. Postharvest Biol. Technol., 92, 79-86.

Cárcel J.A., García-Pérez J.V., Benedito J., Mulet A., 2012. Food process innovation through new technologies: Use of ultrasound. J. Food Eng., 110, 200-207.

Chiralt A. and Talens P., 2005. Physical and chemical changes induced by osmotic dehydration in plant tissues. J. Food Eng., 67, 167-177.

Garcia-Perez J.V., Carcel J.A., de la Fuente-Blanco S., and Riera-Franco de Sarabia E., 2006. Ultrasonic drying of foodstuff in a fluidized bed: Parametric study. Ultrasonics, 44, 539-543.

García-Pérez J.V., Cárcel J.A., Benedito J., and Mulet A., 2007. Power ultrasound mass transfer enhancement in food drying. Trans IChemE, Part C. Food Bioprod. Process, 85(C3), 247-254.

Gerlach D., 1972. Outline of botanical microtechnology (in Polish). PWRiL, Warsaw, Poland.

Harker F.R. and Suthrland P.W., 1993. Physiological changes associated with fruit ripening and the development of mealy texture during storage of nectarines. Postharvest Biol. Technol., 9(4), 269-277.

Ho Q.T., Verboven P., Mebatsion H.K., VerlindenB.E., Vandewalle S., and Nicolaï B.M., 2009. Microscale mechanisms of gas exchange in fruit tissue. New Phytologist, 182, 163-174.

Ho Q.T., Verboven P., Verlinden B.E. , Herremans E.,Wevers M., Carmeliet J., and Nicolaï B.M., 2011. A three-dimensional multiscale model for gas exchange in fruit. Plant Physiol. 155, 1158-1168.

Ho Q.T., Verboven P., Verlinden B.E., and Nicolaï B.M., 2010. A model for gas transport in pear fruit at multiple scales. J. Experimental Bot., 61, 2071-2081.

Kentish S., Ashokkumar M., 2011. The physical and chemical effects of ultrasound. In: Ultrasound Technologies for Food and Bioprocessing (Eds H. G.V. Feng, Barbosa-Canovas, J. Weiss). Springer Press, New York, USA.

Koubaa M., Roselló-Soto E., Šic Žlabur J., Režek Jambrak A., Brnčić M., Grimi N., Boussetta N., and Barba F.J., 2015. Current and new insights in the sustainable and green recovery of nutritionally valuable compounds from Stevia rebaudiana Bertoni. J. Agric. Food Chem., 63, 6835-6846.

Legland D., Devaux M.F., Bouchet B., and Guillon F., 2012. Cartography of cell morphology in tomato pericarp at the fruit scale. J. Microsc., 247(1), 78-93.

Lewicki P.P. and Pawlak-Porzecka R., 2005. Effect of osmotic dewatering on apple tissue structure. J. Food Eng., 66, 43-50.

Mathworks, 2011. Matlab Reference Guide. The MathWorks Inc., Natick, MA, USA. 
Mebatsion H.K., Verboven P., Ho Q.T., Verlinden B.E., and Nicolaï B.M., 2008. Modelling fruit (micro)structures why and how? Trends Food Sci. Technol., 19, 59-66.

Mebatsion H.K., Verboven P., Melese Endalew A., Billen J., Ho Q.T., and Nicolai B.M., 2009. A novel method for 3-D microstructure modeling of pome fruit tissue using synchrotron radiation tomography images. J. Food Eng., 93, 141-148.

Mendoza F., Pieter V., Mebatsion H.K., Kerckhofs G., Wevers M., and Nicolai B., 2007. Three-dimensional pore space quantification of apple tissue using X-ray computed microtomography. Planta, 226, 559-570.

Mothibe K.J., Zhang M., Nsor-atindana J., and Wang Y.C., 2011. Use of ultrasound pretreatment in drying of fruits: Drying rates, quality attributes, and shelf life extension. Drying Technol., 29, 1611-1621.

Nguyen N.D.T., Rizzolo A., Herremans E., Vanolib M., Cortellino G., Erkinbaev C., Tsuta M., Spinelli L., Contini D., Torricelli A., Verboven P., De Baerdemaeker J., Nicolaï B.M., and Saeys W., 2014. Optical properties-microstructure-texture relationships of dried apple slices: Spatially resolved diffuse reflectance spectroscopy as a novel technique for analysis and process control. Innov. Food Sci. Emerg. Technol., 21, 160-168.

Pieczywek P.M. and Zdunek A., 2012. Automatic classification of cells and intercellular spaces of apple tissue. Comput. Electron. Agric., 81, 72-78.

Roselló-Sotoa E., Galanakis C.M., Brnčićc M., Orliend V., Trujilloe F.J., Mawson R., Knoerzer K., Tiwarig B.K., and Barba F.J., 2015a. Clean recovery of antioxidant compounds from plant foods, by-products and algae assisted by ultrasounds processing. Modeling approaches to optimize processing conditions. Trends Food Sci. Technol., 42(2), 134-149.

Rosello-Soto E., Koubaa M., Moubarik A., Lopes R.P., Saraiva J.A., Boussetta N., Grimi N., and Barba F.J., 2015b. Emerging opportunities for the effective valorization of wastes and by-products generated during olive oil production process: Nonconventional methods for the recovery of high-added value compounds. Trends Food Sci. Technol., 45, 296-310.

Šic Žlabur J., Voća S., Dobričević N., Brnčić M., Dujmić F., and Rimac Brnčić S., 2015. Optimization of ultrasound assisted extraction of functional ingredients from Stevia rebaudiana Bertoni leaves. Int. Agrophys., 29, 231-237.

Simal S., Benedito J., Sánchez E.S., and Roselló C., 1998. Use of ultrasound to increase mass transport rate during osmotic dehydration. J. Food Eng., 36, 323-336.

Wan P.J., Muanda M.W., and Covey J.E., 1992. Ultrasonic vs. nonultrasonic hydrogenation in a batch reactor. J. Am. Oil Chem. Soc., 69, 876-879.

Zinoviadou K.G., Galanakis C.M., Brnčić M., Grimi N., Boussetta N., Mota M.J., Saraiva J.A., Patras A., Tiwari B., and Barba F.J., 2015. Fruit juice sonication: Implications on food safety and physicochemical and nutritional properties. Food Research International, http://dx.doi.org/10.1016/ j.foodres.2015.05.032. 